\title{
A WEAK LIMIT THEOREM FOR GENERALIZED JIŘINA PROCESSES
}

\author{
YUQIANG LI,* East China Normal University
}

\begin{abstract}
In this paper we prove that a sequence of scaled generalized Jiřina processes can converge weakly to a nonlinear diffusion process with Lévy jumps under certain conditions.

Keywords: Generalized branching processes; weak convergence; nonlinear diffusion processes
\end{abstract}

2000 Mathematics Subject Classification: Primary 60J80

Secondary 60J60; 60E07

\section{Introduction}

By a generalized Jiřina process we mean a continuous-state population-size-dependent branching process (continuous-state PSDBP) which is a modification of a classical Jiřina process, namely, a continuous-state branching process with discrete time [5], taking into account the fact that the reproductive behavior may depend on the size of the population. Here we recall its definition.

A time-homogeneous Markov process $\{Y(k), k=0,1,2, \ldots\}$ with state space $[0, \infty)$ is called a continuous-state PSDBP if its one-step transition function $P(x, \mathrm{~d} y)$ satisfies

$$
\int_{[0, \infty)} \mathrm{e}^{-\lambda y} P(x, \mathrm{~d} y)=\exp \left\{-x\left(\gamma(x) \lambda+\int_{(0, \infty)}\left(1-\mathrm{e}^{-\lambda u}\right) v(x, \mathrm{~d} u)\right)\right\} .
$$

Here $\gamma(x)$ is a nonnegative Borel function and $(1 \wedge u) v(x, \mathrm{~d} u)$ is a finite kernel from $[0, \infty)$ to $(0, \infty)$.

Obviously, a continuous-state PSDBP is determined by the pair of functions $\gamma(x)$ and $v(x, \cdot)$.

For any $x \geq 0$, define $m(x):=\gamma(x)+\int_{(0, \infty)} u v(x, \mathrm{~d} u)$ and $\sigma^{2}(x):=\int_{(0, \infty)} u^{2} v(x, \mathrm{~d} u)$. We call $m(x)$ and $\sigma^{2}(x)$ the offspring mean and the offspring variance (when the parent population is of size $x$ ), respectively, if the corresponding integral is finite. When $m(x)$ and $\sigma(x)$ are finite for all $x$, it is easy to obtain, for any $k>0$,

$$
\mathrm{E}[Y(k) \mid Y(k-1)]=m(Y(k-1)) Y(k-1)
$$

and

$$
\mathrm{E}\left[(Y(k)-m Y(k-1))^{2} \mid Y(k-1)\right]=\sigma^{2}(Y(k-1)) Y(k-1) .
$$

The continuous-state PSDBP was first introduced by Li [9], who showed that it can arise from the limit of a sequence of suitably scaled PSDBPs with discrete states [3], [8].

Diffusion approximation for branching processes was formulated by Feller [2] in 1951. He described a procedure for obtaining diffusions as limits of Galton-Watson processes.

Received 7 February 2008; revision received 23 March 2009.

* Postal address: School of Finance and Statistics, East China Normal University, Shanghai 200241, China.

Email address: yqli@stat.ecnu.edu.cn 
Jiřina [6] gave a more precise proof of Feller's assertion. Since then, many authors have done much work in this field. See, for example, [4], [11], and the references therein.

In 1977, Lipow [13] studied the diffusion approximation for state-dependent branching processes. He considered a sequence of continuous-time discrete-state branching processes $\left\{Z_{n}(t)\right\}, n=1,2, \ldots$, whose reproductive behavior depends on the size of the population. Using Kurtz's theorem for the convergence of a semigroup, Lipow proved that, under certain conditions, the sequence $\left\{Z_{n}(n t) / n, t \geq 0\right\}, n=1,2, \ldots$, converges weakly to a diffusion process with generator

$$
\mathcal{A} f(x)=\lambda x\left[\beta f^{\prime \prime}(x)+\alpha(x) f^{\prime}(x)\right],
$$

where $\lambda$ and $\beta$ are positive constants, and $\alpha(x)$ is a bounded continuous function on $[0, \infty)$.

In addition, by means of a semigroup, Rosenkranz [14] showed that, under certain conditions, a sequence of density-dependent branching processes with random environment converges weakly to a diffusion process which can be obtained as a solution of a stochastic differential equation.

Motivated by their work, in this paper we discuss the relation between continuous-state PSDBPs and diffusion processes. More precisely, we are interested in the relation between the continuous-state PSDBPs and the diffusion processes with Lévy generators $\mathcal{L}$ satisfying

$$
\mathcal{L} f(x)=x \alpha(x) f^{\prime}(x)+x \beta(x) f^{\prime \prime}(x)+x \int_{(0, \infty)}\left(f(x+u)-f(x)-f^{\prime}(x) u\right) \mu(x, \mathrm{~d} u)
$$

for any $f \in C_{c}^{\infty}[0, \infty)$, where $\alpha(x)$ and $\beta(x)$ are two functions, and $C_{c}^{\infty}[0, \infty)$ is the set of all infinite differentiable functions $f:[0, \infty) \rightarrow(-\infty, \infty)$ with compact support.

This kind of problem was also considered by Kawazu and Watanabe [7]. In [7], a continuousstate branching process (CSBP) and a CSBP with immigration, when immigration components exist, were defined. They pointed out that a conservative CSBP with immigration has the following generator:

$$
\begin{aligned}
\mathcal{A} f(x)= & a x f^{\prime \prime}(x)+(b x+d) f^{\prime}(x)+\int_{(0, \infty)}(f(x+y)-f(x)) v_{1}(\mathrm{~d} y) \\
& +x \int_{(0, \infty)}\left(f(x+y)-f(x)-\frac{y}{1+y^{2}} f^{\prime}(x)\right) v(\mathrm{~d} y),
\end{aligned}
$$

and that a Markov process with the above generator is a CSBP with immigration, where $a \geq 0$, $b$, and $d \geq 0$ are constants, and $v_{1}$ and $v$ are two nonnegative measures on $(0, \infty)$ such that

$$
\int_{(0, \infty)} \frac{y^{2}}{1+y^{2}} v(\mathrm{~d} y)+\int_{(0, \infty)} \frac{y}{1+y} v_{1}(\mathrm{~d} y)<\infty .
$$

Then, Kawazu and Watanabe [7] proved that, under mild conditions, a sequence of scaled Galton-Watson processes with immigrations converges in finite-dimensional distributions to a CSBP with immigration. In addition, Li [12] extended this result from the convergence of finite-dimensional distributions to weak convergence in the Skorokhod space $D_{[0, \infty)}[0, \infty)$, namely, the space of càdlàg functions (i.e. those that are right continuous with left limits) from $[0, \infty)$ to $[0, \infty)$ with Skorokhod topology.

From [7] and [12] we can readily find that if $\int_{(0, \infty)}\left(y \wedge y^{2}\right) v(\mathrm{~d} y)<\infty$ then the generator of the conservative CSBP can be written as

$$
\mathcal{A} f(x)=a x f^{\prime \prime}(x)+\bar{b} x f^{\prime}(x)+x \int_{(0, \infty)}\left(f(x+y)-f(x)-y f^{\prime}(x)\right) v(\mathrm{~d} y)
$$


for some $\bar{b} \geq b$, and that, under mild conditions, a sequence of scaled Galton-Watson processes converges weakly in $D_{[0, \infty)}[0, \infty)$ to a CSBP. For the similarity between $\mathcal{L}$ and $\mathcal{A}$, we naturally regard the diffusion process $X$ with Lévy generator $\mathcal{L}$ as a generalized CSBP. Hence, the work of the present paper can be seen as a generalization of that of [7] and [12] in some sense. It is proved in the present paper that, under certain conditions, a sequence of scaled continuous-state PSDBPs converges weakly in the Skorokhod space $D_{[0, \infty)}[0, \infty)$ to a generalized CSBP.

The main tool used in this paper is the convergence theory of martingale problems; see [1, Chapter 4, Corollary 8.17]). Below, we briefly introduce some basic definitions on martingale problems. For further details, we refer the reader to [1, Chapter 3].

For a metric space $E, D_{E}[0, \infty)$ denotes the Skorokhod space of càdlàg functions from $[0, \infty)$ to $E$. Let $\mathcal{T}$ be an operator from $D(\mathcal{T}) \subset B(E)$ to $B(E)$, where $B(E)$ is the collection of bounded measurable functions on $E$ and $D(\mathcal{T})$ is the domain of $\mathcal{T}$. By a solution of the martingale problem for $\mathcal{T}$ we mean a measurable stochastic process $X$ with value in $E$ defined on some probability space $(\Omega, \mathcal{F}, \mathrm{P})$ such that, for each $f \in D(\mathcal{T})$,

$$
f(X(t))-\int_{0}^{t} \mathcal{T} f(X(s)) \mathrm{d} s
$$

is a martingale with respect to the filtration

$$
* \mathcal{F}_{t}^{X}=\mathcal{F}_{t}^{X} \vee \sigma\left(\int_{0}^{s} h(X(u)) \mathrm{d} u: s \leq t, h(\cdot) \in B(E)\right),
$$

where $\mathcal{F}_{t}^{X}=\sigma(X(s), 0 \leq s \leq t)$. We say that a solution $X$ of the martingale problem for $\mathcal{T}$ is a solution of the martingale problem for $(\mathcal{T}, x)$ in $D_{E}[0, \infty)$ if $X(0)=x$ almost surely and $X$ is right continuous with left limits in path. We say that the uniqueness holds for solutions of the martingale problem for $(\mathcal{T}, x)$ in $D_{E}[0, \infty)$ if any two solutions in $D_{E}[0, \infty)$ have the same finite-dimensional distributions. If there exists a solution of the martingale problem for $(\mathcal{T}, x)$ in $D_{E}[0, \infty)$ and the uniqueness holds, we say that the martingale problem for $(\mathcal{T}, x)$ is well posed in $D_{E}[0, \infty)$.

This paper is organized as follows. In Section 2 we introduce the main assumptions and results of this paper. The proofs of the results are given in Section 3.

\section{The main results}

In the sequel, unless otherwise stated, let $\mathcal{L}$ be the operator defined in (1.3). Furthermore, we suppose that the following conditions hold.

(H1) $x \beta(x)$ and $\beta(x)$ are bounded and continuous and $\beta(x)>0$ for $x>0$.

(H2) $x \alpha(x)$ and $\alpha(x)$ are bounded and continuous.

(H3) For any Borel measurable set $\Gamma \subset(0, \infty), \int_{\Gamma} u^{2} x \mu(x, \mathrm{~d} u)$ is bounded and continuous.

(H4) $b(x):=\int_{(0, \infty)} u^{2} \mu(x, \mathrm{~d} u)$ is bounded and continuous.

Let $\left\{Y_{n}(k), k \geq 0\right\}_{n}$ be a sequence of continuous-state PSDBPs given by a sequence of parameters $\gamma_{n}(x)$ and $v_{n}(x, \cdot)$. The corresponding offspring mean and offspring variance are respectively denoted by $m_{n}(x)$ and $\sigma_{n}^{2}(x)$. We assume the following conditions.

(E1) For $x \geq 0, m_{n}(x)=1+\alpha_{n}(x) / n>0$ and $\sigma_{n}^{2}(x)=\beta_{n}(x) / n>0$, where $\alpha_{n}(x)$ and $\beta_{n}(x)$ are uniformly bounded. 
(E2) $\alpha_{n}(x)$ and $\beta_{n}(x)$ converge locally uniformly to continuous functions $\alpha(x)$ and $2 \beta(x)+$ $b(x)$, respectively.

(E3) For $x \in[0, \infty)$, let $\mu_{n}(x, \cdot)=v_{n}(x, \cdot)-n^{-1} \mu(x, \cdot)$ and $\tau_{n}(x)=\int_{(0, \infty)} u^{3}\left|\mu_{n}\right|(x, \mathrm{~d} u)$, where $\left|\mu_{n}\right|(x, \cdot)$ is the total variation measure of $\mu_{n}(x, \cdot)$. Then $n \tau_{n}(x)$ converges locally uniformly to 0 .

The fact that a sequence of functions $T_{n}(x)$ converges locally uniformly to a function $T(x)$ means that, for any bounded set $I \subset[0, \infty), \lim _{n \rightarrow+\infty} \sup _{x \in I}\left|T_{n}(x)-T(x)\right|=0$.

We have the following result for

$$
X_{n}(t)=Y_{n}([n t]),
$$

where $[n t]$ is the largest integer bounded by $n t$.

Theorem 2.1. Suppose that (H1)-(H4) and (E1)-(E3) hold. Let $Y_{n}(0) \equiv x_{0} \geq 0$. Then there exists a solution $X$ of the martingale problem for $\left(\mathcal{L}, x_{0}\right)$ such that $X_{n}$ converges weakly to $X$ in the Skorokhod space $D_{[0, \infty)}[0, \infty)$.

The converse of Theorem 2.1 holds in some sense.

Theorem 2.2. Suppose that (H1)-(H4) hold and that $X(t)$ is the unique solution of the martingale problem for $\left(\mathcal{L}, x_{0}\right)$, where $x_{0} \geq 0$. Furthermore, we assume that $a(x):=\int_{(0, \infty)} u \mu(x, \mathrm{~d} u)$ is a bounded continuous function. Then there exists a sequence of continuous-state PSDBPs $Y_{n}$ satisfying assumptions $(E 1)-(E 3)$ and $Y_{n}(0)=x_{0}$. Hence, there exists a version of $X(t)$ such that $X_{n}(t)=Y_{n}([n t])$ converges weakly in $D_{[0, \infty)}[0, \infty)$ to this version.

Remark 2.1. Theorem 2.2 still shows that conditions (E1)-(E3) are meaningful.

We will prove Theorem 2.1 via Corollary 8.17 of [1, Chapter 4], which requires two preconditions. One is the uniqueness of the solutions to the martingale problem for $\left(\mathcal{L}, x_{0}\right)$ in $D_{[0, \infty)}[0, \infty)$. This work was done in [10] by a standard method; see [10, Theorem 2.1]. In fact, based on Theorem 4.3 of [15], by the stopping time arguments, we can readily conceive that, under conditions (H1)-(H4), the martingale problem for $\left(\mathcal{L}, x_{0}\right)$ is well posed in $D_{[0, \infty)}[0, \infty)$. The other requirement is that the sequence $X_{n}$ satisfies the compact containment condition in $D_{[0, \infty)}[0, \infty)$. We have the following lemma.

Lemma 2.1. Let $\left\{Y_{n}\right\}$ be a sequence of continuous-state PSDBPs with $0<m_{n}(x)=1+$ $\alpha_{n}(x) / n$. Suppose that there exists a constant $\xi>0$ such that $\alpha_{n}(x)<\xi$ for all $n$ and $x$. Then $\left\{X_{n}\right\}$ defined by (2.1) satisfies the compact containment condition.

From this lemma we immediately obtain the compact containment condition for $X_{n}$.

Corollary 2.1. Under the assumptions of Theorem 2.1, $\left\{X_{n}\right\}$ satisfies the compact containment condition.

The proofs of Theorem 2.1, Theorem 2.2, and Lemma 2.1 are given in the next section. For convenience, let $Z_{n}(x):=Y_{n}(1)-x$ and $\mathrm{E}_{x}\left[f\left(Y_{n}(1)\right)\right]:=\mathrm{E}\left[f\left(Y_{n}(1)\right) \mid Y_{n}(0)=x\right]$.

\section{The proofs of the main results}

Proof of Lemma 2.1. The proof is equal to checking that, for any $\eta>0$ and $T \geq 0$, there exists a compact set $\Gamma_{\eta, T} \subset[0, \infty)$ such that

$$
\liminf _{n \rightarrow \infty} \mathrm{P}\left\{X_{n}(t) \in \Gamma_{\eta, T} \text { for } 0 \leq t \leq T\right\} \geq 1-\eta .
$$


Define a compact set $\Gamma_{\eta, T}:=\left[0,1 / \eta \mathrm{e}^{\xi T} x_{0}\right]$. Let $M_{n}(t):=X_{n}(t) / \sum_{i=0}^{[n t]-1} m_{n}\left(Y_{n}(i)\right)$. Then, for any $n=1,2, \ldots, M_{n}$ is a martingale. Observe that

$$
\begin{aligned}
& \mathrm{P}\left\{X_{n}(t) \in \Gamma_{\eta, T} \text { for } 0 \leq t \leq T\right\} \\
& \quad=1-\mathrm{P}\left\{M_{n}(t)>\frac{1}{\eta} \mathrm{e}^{\xi T} x_{0} / \sum_{i=0}^{[n t]-1} m_{n}\left(Y_{n}(i)\right) \text { for some } 0 \leq t \leq T\right\} \\
& \quad \geq 1-\mathrm{P}\left\{\sup _{0 \leq t \leq T} M_{n}(t)>\frac{\mathrm{e}^{\xi T} x_{0}}{\eta(1+\xi / n)^{[n T]}}\right\} .
\end{aligned}
$$

Doob's inequality indicates that

$$
\mathrm{P}\left\{\sup _{0 \leq t \leq T} M_{n}(t)>\frac{\mathrm{e}^{\xi T} x_{0}}{\eta(1+\xi / n)^{[n T]}}\right\} \leq \frac{\eta(1+\xi / n)^{[n T]}}{\mathrm{e}^{\xi T} x_{0}} \sup _{0 \leq t \leq T} \mathrm{E}\left[M_{n}(t)\right]=\frac{\eta(1+\xi / n)^{[n T]}}{\mathrm{e}^{\xi T}} .
$$

Hence, (3.2) implies that

$$
\mathrm{P}\left\{X_{n}(t) \in \Gamma_{\eta, T} \text { for } 0 \leq t \leq T\right\} \geq 1-\frac{\eta(1+\xi / n)^{[n T]}}{\mathrm{e}^{\xi T}} .
$$

Then (3.1) follows from (3.3) as $n \rightarrow \infty$. This completes the proof.

Proof of Theorem 2.1. According to Corollary 8.17 of [1, Chapter 4], it suffices to prove that

$$
\lim _{n \rightarrow+\infty} \sup _{x \in[0, \infty)}\left|\mathcal{A}_{n} f(x)-\mathcal{L} f(x)\right|=0
$$

for any $f \in C_{c}^{\infty}[0, \infty)$, where $\mathcal{A}_{n} f(x)=n\left(\mathrm{E}_{x}\left[f\left(Y_{n}(1)\right)\right]-f(x)\right)$. To this end, it is enough to prove that, for any $x_{n} \in[0, \infty)$, if $x_{n} \rightarrow x \in[0, \infty]$ then

$$
\lim _{n \rightarrow+\infty}\left(\mathcal{A}_{n} f\left(x_{n}\right)-\mathcal{L} f\left(x_{n}\right)\right)=0
$$

for any given $f \in C_{c}^{\infty}[0, \infty)$.

From (1.1), it follows that

$$
n \mathrm{E}_{x_{n}}\left[f^{\prime}\left(x_{n}\right) Z_{n}\left(x_{n}\right)\right]=n x_{n} f^{\prime}\left(x_{n}\right)\left(m_{n}\left(x_{n}\right)-1\right)=x_{n} \alpha_{n}\left(x_{n}\right) f^{\prime}\left(x_{n}\right) .
$$

By Taylor's expansion we obtain

$$
\begin{aligned}
\mathcal{A}_{n} f\left(x_{n}\right) & =n \mathrm{E}_{x_{n}}\left[f\left(Y_{n}(1)\right)-f\left(x_{n}\right)\right] \\
& =n \mathrm{E}_{x_{n}}\left[f^{\prime}\left(x_{n}\right) Z_{n}\left(x_{n}\right)\right]+\Delta_{n}\left(x_{n}\right) \\
& =x_{n} \alpha_{n}\left(x_{n}\right) f^{\prime}\left(x_{n}\right)+\Delta_{n}\left(x_{n}\right),
\end{aligned}
$$

where

$$
\Delta_{n}\left(x_{n}\right)=\mathrm{E}_{x_{n}}\left[n \int_{0}^{1}(1-w) f^{\prime \prime}\left(x_{n}+w Z_{n}\left(x_{n}\right)\right) Z_{n}^{2}\left(x_{n}\right) \mathrm{d} w\right] .
$$

In the remainder of the proof, we consider two cases.

Case 1: $x_{n} \rightarrow x=+\infty$. In this case, for sufficiently large $n$, we have $x_{n}>\delta$, where $\delta$ is an upper bound of the support set of $f \in C_{c}^{\infty}[0, \infty)$. Therefore, for sufficiently large $n$, $\mathcal{L} f\left(x_{n}\right)=0$ and $x_{n} \alpha_{n}\left(x_{n}\right) f^{\prime}\left(x_{n}\right)=0$. Consequently, (3.4) is equivalent to

$$
\lim _{n \rightarrow \infty} \Delta_{n}\left(x_{n}\right)=0 \text {. }
$$


Observe that

$$
x_{n}+w Z_{n}\left(x_{n}\right)=x_{n}+w\left(Y_{n}(1)-x_{n}\right) \geq(1-w) x_{n} .
$$

The integrand in $\Delta_{n}\left(x_{n}\right)$ is 0 if $w<1-\delta / x_{n}$. Hence, from (1.2), it follows that

$$
\begin{aligned}
\Delta_{n}\left(x_{n}\right) & \leq \mathrm{E}_{x_{n}}\left[\int_{1-\delta / x_{n}}^{1}(1-w)\left\|f^{\prime \prime}\right\| n Z_{n}^{2}\left(x_{n}\right) \mathrm{d} w\right] \\
& =\frac{\delta^{2}}{2 x_{n}^{2}} n \mathrm{E}_{x_{n}}\left[Z_{n}^{2}\left(x_{n}\right)\right] \\
& =\frac{\delta^{2}}{2 x_{n}^{2}}\left(n\left(m_{n}\left(x_{n}\right)-1\right)^{2} x_{n}^{2}+n \sigma_{n}^{2}\left(x_{n}\right) x_{n}\right) \\
& =\frac{\delta^{2}}{2}\left(\frac{\alpha_{n}^{2}\left(x_{n}\right)}{n}+\frac{\beta_{n}\left(x_{n}\right)}{x_{n}}\right) .
\end{aligned}
$$

From assumption (E1) we have

$$
\lim _{n \rightarrow \infty}\left(\frac{\alpha_{n}^{2}\left(x_{n}\right)}{n}+\frac{\beta_{n}\left(x_{n}\right)}{x_{n}}\right)=0 .
$$

Then (3.5) follows from (3.6) and (3.7).

Case 2: $x_{n} \rightarrow x<+\infty$. In this case, by (H2) and (E2), we can readily obtain

$$
x_{n} f^{\prime}\left(x_{n}\right) \alpha_{n}\left(x_{n}\right) \rightarrow x \alpha(x) f^{\prime}(x) \text { as } n \rightarrow \infty .
$$

At the same time, (H3) and (H4) imply that

$$
\begin{aligned}
& \mid \int_{(0, \infty)}\left(f\left(x_{n}+u\right)-f\left(x_{n}\right)-f^{\prime}\left(x_{n}\right) u\right) x_{n} \mu\left(x_{n}, \mathrm{~d} u\right) \\
& -\int_{(0, \infty)}\left(f(x+u)-f(x)-f^{\prime}(x) u\right) x_{n} \mu\left(x_{n}, \mathrm{~d} u\right) \mid \\
& \quad \leq \int_{(0, \infty)}\left|f^{\prime}\left(z_{n}+u\right)-f^{\prime}\left(z_{n}\right)-f^{\prime \prime}\left(z_{n}\right) u\right|\left|x_{n}-x\right| x_{n} \mu\left(x_{n}, \mathrm{~d} u\right) \\
& \quad \leq\left\|f^{\prime \prime \prime}\right\|\left|x_{n}-x\right| \int_{(0, \infty)} u^{2} x_{n} \mu\left(x_{n}, \mathrm{~d} u\right) \\
& \quad \rightarrow 0
\end{aligned}
$$

where $z_{n} \in\left(x, x_{n}\right)$. Let $\phi_{x}(u)=\left(f(x+u)-f(x)-f^{\prime}(x) u\right) / u^{2}$ for $u \in(0, \infty)$. From $f \in C_{c}^{\infty}[0, \infty)$ we know that $\phi_{x}(u)$ is bounded and continuous for any $x \geq 0$. Therefore, $(\mathrm{H} 3)$ indicates that, as $n \rightarrow \infty$,

$$
\begin{aligned}
& \int_{(0, \infty)}\left(f(x+u)-f(x)-f^{\prime}(x) u\right) x_{n} \mu\left(x_{n}, \mathrm{~d} u\right) \\
& -\int_{(0, \infty)}\left(f(x+u)-f(x)-f^{\prime}(x) u\right) x \mu(x, \mathrm{~d} u), \\
& \quad=\int_{(0, \infty)} \phi_{x}(u) x_{n} u^{2} \mu\left(x_{n}, \mathrm{~d} u\right)-\int_{(0, \infty)} \phi_{x}(u) x u^{2} \mu(x, \mathrm{~d} u) \\
& \quad \rightarrow 0 .
\end{aligned}
$$


Combining (3.8) and (3.9), we obtain

$$
\begin{aligned}
\int_{(0, \infty)} & \left(f\left(x_{n}+u\right)-f\left(x_{n}\right)-f^{\prime}\left(x_{n}\right) u\right) x_{n} \mu\left(x_{n}, \mathrm{~d} u\right) \\
\rightarrow & \int_{(0, \infty)}\left(f(x+u)-f(x)-f^{\prime}(x) u\right) x \mu(x, \mathrm{~d} u),
\end{aligned}
$$

which implies that $\mathcal{L} f\left(x_{n}\right) \rightarrow \mathcal{L} f(x)$ as $x_{n} \rightarrow x<\infty$. Consequently, to prove (3.4), it is enough to prove that

$$
\Delta_{n}\left(x_{n}\right) \rightarrow \beta(x) x f^{\prime \prime}(x)+\int_{(0, \infty)}\left(f(x+u)-f(x)-f^{\prime}(x) u\right) x \mu(x, \mathrm{~d} u),
$$

which is proved in the following lemma.

Lemma 3.1. Using the same assumptions and notation as in Theorem 2.1 and its proof, if $x_{n} \rightarrow x<\infty$ then (3.10) holds.

Proof. By Fubini's theorem we have

$$
\begin{aligned}
\Delta_{n}\left(x_{n}\right)= & \int_{0}^{1}(1-w) \mathrm{E}_{x_{n}}\left[n f^{\prime \prime}\left(x_{n}+w Z_{n}\left(x_{n}\right)\right) Z_{n}^{2}\left(x_{n}\right)\right] \mathrm{d} w \\
& -\int_{0}^{1}(1-w) \mathrm{E}_{x_{n}}\left[n f^{\prime \prime}\left(x+w Z_{n}\left(x_{n}\right)\right) Z_{n}^{2}\left(x_{n}\right)\right] \mathrm{d} w \\
& +\int_{0}^{1}(1-w) \mathrm{E}_{x_{n}}\left[n f^{\prime \prime}\left(x+w Z_{n}\left(x_{n}\right)\right) Z_{n}^{2}\left(x_{n}\right)\right] \mathrm{d} w
\end{aligned}
$$

Since $f^{\prime \prime}$ is uniformly continuous, the dominated convergence theorem implies that, as $x_{n} \rightarrow x$,

$$
\begin{aligned}
& \int_{0}^{1}(1-w) \mathrm{E}_{x_{n}}\left[n f^{\prime \prime}\left(x_{n}+w Z_{n}\left(x_{n}\right)\right) Z_{n}^{2}\left(x_{n}\right)\right] \mathrm{d} w \\
& -\int_{0}^{1}(1-w) \mathrm{E}_{x_{n}}\left[n f^{\prime \prime}\left(x+w Z_{n}\left(x_{n}\right)\right) Z_{n}^{2}\left(x_{n}\right)\right] \mathrm{d} w \rightarrow 0 .
\end{aligned}
$$

Hence, we only need to prove that

$$
\begin{aligned}
\int_{0}^{1}(1-w) \mathrm{E}_{x_{n}}\left[n f^{\prime \prime}\left(x+w Z_{n}\left(x_{n}\right)\right) Z_{n}^{2}\left(x_{n}\right)\right] \mathrm{d} w \\
\quad \rightarrow \beta(x) x f^{\prime \prime}(x)+\int_{(0, \infty)}\left(f(x+u)-f(x)-f^{\prime}(x) u\right) x \mu(x, \mathrm{~d} u) .
\end{aligned}
$$

It suffices to prove that, as $x_{n} \rightarrow x$,

$$
\begin{aligned}
\mathrm{E}_{x_{n}}\left[n f^{\prime \prime}\left(x+w Z_{n}\left(x_{n}\right)\right) Z_{n}^{2}\left(x_{n}\right)\right] & \\
& \rightarrow 2 x \beta(x) f^{\prime \prime}(x)+x \int_{(0, \infty)} f^{\prime \prime}(x+w u) u^{2} \mu(x, \mathrm{~d} u) .
\end{aligned}
$$

In fact, if (3.12) holds then (3.11) follows from Taylor's expansion. 
Let $M_{x}=-\sup _{n} x_{n}$. For any $n \geq 0$, define a measure $Q_{n}(\cdot)$ on $\left[M_{x}, \infty\right)$ such that, for any Borel measurable set $A \subset\left[M_{x}, \infty\right)$,

$$
Q_{n}(A):=\mathrm{E}_{x_{n}}\left[n \mathbf{1}_{A}\left(Z_{n}\left(x_{n}\right)\right) Z_{n}^{2}\left(x_{n}\right)\right]=\mathrm{E}_{x_{n}}\left[n \mathbf{1}_{A}\left(Y_{n}(1)-x_{n}\right)\left(Y_{n}(1)-x_{n}\right)^{2}\right] .
$$

Then, for any $\lambda \geq 0$,

$$
\int_{\left[M_{x}, \infty\right)} \mathrm{e}^{-\lambda u} Q_{n}(\mathrm{~d} u)=\mathrm{E}_{x_{n}}\left[n \exp \left\{-\lambda\left(Y_{n}(1)-x_{n}\right)\right\}\left(Y_{n}(1)-x_{n}\right)^{2}\right] .
$$

Note that $Y_{n}$ is the continuous-state PSDBP with parameters $\gamma_{n}(x)$ and $v_{n}(x, \cdot)$. We have

$$
\begin{aligned}
\mathrm{E}_{x_{n}} & {\left[\exp \left\{-\lambda\left(Y_{n}(1)-x_{n}\right)\right\}\right] } \\
& =\exp \left\{-x_{n}\left(\left(\gamma_{n}\left(x_{n}\right)-1\right) \lambda+\int_{(0, \infty)}\left(1-\mathrm{e}^{-\lambda u}\right) v_{n}\left(x_{n}, \mathrm{~d} u\right)\right)\right\} \\
& =\exp \left\{-x_{n}\left(\left(m_{n}\left(x_{n}\right)-1\right) \lambda+\int_{(0, \infty)}\left(1-\mathrm{e}^{-\lambda u}-\lambda u\right) v_{n}\left(x_{n}, \mathrm{~d} u\right)\right)\right\} .
\end{aligned}
$$

Using this formula, via some simple calculation, we obtain

$$
\begin{aligned}
\mathrm{E}_{x_{n}}[ & \left.n \exp \left\{-\lambda\left(Y_{n}(1)-x_{n}\right)\right\}\left(Y_{n}(1)-x_{n}\right)^{2}\right] \\
& =\psi\left(n, x_{n}, \lambda\right) \phi\left(n, x_{n}, \lambda\right)+n \varphi\left(n, x_{n}, \lambda\right)^{2} \phi\left(n, x_{n}, \lambda\right),
\end{aligned}
$$

where

$$
\begin{aligned}
& \phi\left(n, x_{n}, \lambda\right)=\exp \left\{-x_{n}\left(\left(m_{n}\left(x_{n}\right)-1\right) \lambda+\int_{(0, \infty)}\left(1-\mathrm{e}^{-\lambda u}-\lambda u\right) v_{n}\left(x_{n}, \mathrm{~d} u\right)\right)\right\} \\
& \varphi\left(n, x_{n}, \lambda\right)=-x_{n}\left(m_{n}\left(x_{n}\right)-1+\int_{(0, \infty)} u\left(\mathrm{e}^{-\lambda u}-1\right) v_{n}\left(x_{n}, \mathrm{~d} u\right)\right) \\
& \psi\left(n, x_{n}, \lambda\right)=x_{n} \int_{(0, \infty)} u^{2} \mathrm{e}^{-\lambda u} \mu\left(x_{n}, \mathrm{~d} u\right)+x_{n} \int_{(0, \infty)} u^{2} \mathrm{e}^{-\lambda u} n \mu_{n}\left(x_{n}, \mathrm{~d} u\right) .
\end{aligned}
$$

Note that

$$
\left|-x_{n}\left(\left(m_{n}\left(x_{n}\right)-1\right) \lambda+\int_{(0, \infty)}\left(1-\mathrm{e}^{-\lambda u}-\lambda u\right) v_{n}\left(x_{n}, \mathrm{~d} u\right)\right)\right| \leq \lambda \frac{x_{n}\left|\alpha_{n}\left(x_{n}\right)\right|}{n}+\lambda^{2} \frac{\beta_{n}\left(x_{n}\right)}{n}
$$

and that

$$
\left|\varphi\left(n, x_{n}, \lambda\right)\right| \leq x_{n} \frac{\left|\alpha_{n}\left(x_{n}\right)\right|+\lambda \beta_{n}\left(x_{n}\right)}{n} .
$$

Then by condition (A1) we have, as $n \rightarrow+\infty$,

$$
\phi\left(n, x_{n}, \lambda\right) \rightarrow 1, \quad n \varphi\left(n, x_{n}, \lambda\right)^{2} \rightarrow 0 .
$$

Since

$$
\int_{(0, \infty)} u^{2} \mu_{n}(x, \mathrm{~d} u)=\frac{\beta_{n}(x)-b(x)}{n}
$$

(E3) implies that

$$
\left|\int_{(0, \infty)} u^{2} \mathrm{e}^{-\lambda u} n \mu_{n}\left(x_{n}, \mathrm{~d} u\right)-\left(\beta_{n}\left(x_{n}\right)-b\left(x_{n}\right)\right)\right| \leq \lambda \int_{(0, \infty)} u^{3} n\left|\mu_{n}\right|\left(x_{n}, \mathrm{~d} u\right) \rightarrow 0 .
$$


Furthermore, from (E2)-(E3) and (H1)-(H2), it follows that $\beta_{n}\left(x_{n}\right)-b\left(x_{n}\right) \rightarrow 2 \beta(x)$. Hence,

$$
\psi\left(n, x_{n}, \lambda\right) \rightarrow x \int_{(0, \infty)} u^{2} \mathrm{e}^{-\lambda s} \mu(x, \mathrm{~d} u)+2 x \beta(x) .
$$

Combining (3.15) with (3.16) and (3.17), we obtain

$$
\mathrm{E}_{x_{n}}\left[n \exp \left\{-\lambda\left(Y_{n}(1)-x_{n}\right)\right\}\left(Y_{n}(1)-x_{n}\right)^{2}\right] \rightarrow x \int_{(0, \infty)} u^{2} \mathrm{e}^{-\lambda s} \mu(x, \mathrm{~d} u)+2 x \beta(x) .
$$

Define a measure $\bar{\mu}(x, \cdot)$ on $\left[M_{x}, \infty\right)$ as follows:

$$
\bar{\mu}(x, A)= \begin{cases}\int_{(0, \infty) \cap A} x u^{2} \mu(x, \mathrm{~d} u)+2 x \beta(x), & 0 \in A, \\ \int_{(0, \infty) \cap A} x u^{2} \mu(x, \mathrm{~d} u), & \text { otherwise, }\end{cases}
$$

for any Borel measurable set $A \subset\left[M_{x}, \infty\right)$. Equations (3.13), (3.14) and (3.18) imply that

$$
\int_{\left[M_{x}, \infty\right)} \mathrm{e}^{-\lambda u} Q_{n}(\mathrm{~d} u) \rightarrow \int_{\left[M_{x}, \infty\right)} \mathrm{e}^{-\lambda u} \bar{\mu}(\mathrm{d} u) \quad \text { as } n \rightarrow+\infty .
$$

Hence, for any bounded continuous function $h(u)$ on $\left[M_{x}, \infty\right)$,

$$
\begin{aligned}
\mathrm{E}_{x_{n}}\left[n h\left(Y_{n}(1)-x_{n}\right)\left(Y_{n}(1)-x_{n}\right)^{2}\right] & =\int_{\left[M_{x}, \infty\right)} h(u) Q_{n}(\mathrm{~d} u) \\
& \rightarrow \int_{\left[M_{x}, \infty\right)} h(u) \bar{\mu}(\mathrm{d} u) \\
& =2 x \beta(x) h(0)+x \int_{(0, \infty)} h(u) u^{2} \mu(x, \mathrm{~d} u) .
\end{aligned}
$$

Let $h(u)=f^{\prime \prime}(x+w u)$. Then (3.12) holds.

Proof of Theorem 2.2. From the assumptions of Theorem 2.2, for any $n \geq 1$, we can construct a continuous-state PSDBP $Y_{n}$ which satisfies

$$
\begin{aligned}
& \mathrm{E}\left[\mathrm{e}^{-\lambda Y_{n}(k+1)} \mid Y_{n}(k)=x\right] \\
& \quad=\exp \left\{-x\left(\frac{\mathrm{e}^{(\alpha(x)-a(x)) / n} \lambda}{1+n^{-1} \lambda \beta(x)}+\frac{1}{n} \int_{(0, \infty)}\left(1-\mathrm{e}^{-\lambda u}\right) \mu(x, \mathrm{~d} u)\right)\right\} \\
& \quad=\exp \left\{-x\left(\gamma_{n}(x) \lambda+\int_{(0, \infty)}\left(1-\mathrm{e}^{-\lambda u}\right) \mu_{n}(x, \mathrm{~d} u)+\frac{1}{n} \int_{(0, \infty)}\left(1-\mathrm{e}^{-\lambda u}\right) \mu(x, \mathrm{~d} u)\right)\right\}
\end{aligned}
$$

for any $\lambda \geq 0$, where

$$
\begin{gathered}
\gamma_{n}(x)= \begin{cases}0, & \beta(x)>0, \\
\mathrm{e}^{(\alpha(x)-a(x)) / n}, & \beta(x)=0,\end{cases} \\
\mu_{n}(x, \mathrm{~d} u)= \begin{cases}\mathrm{e}^{(\alpha(x)-a(x)) / n}\left(\frac{n}{\beta(x)}\right)^{2} \mathrm{e}^{-n u / \beta(x)} \mathrm{d} u, & \beta(x)>0, \\
0, & \beta(x)=0 .\end{cases}
\end{gathered}
$$


By some simple calculation, we obtain

$$
m_{n}(x)=\mathrm{e}^{(\alpha(x)-a(x)) / n}+\frac{a(x)}{n}, \quad \sigma_{n}^{2}(x)=\mathrm{e}^{(\alpha(x)-a(x)) / n} \frac{2 \beta(x)}{n}+\frac{b(x)}{n},
$$

and

$$
\tau_{n}(x):=\int_{(0, \infty)} u^{3} \mu_{n}(x, \mathrm{~d} u)=6 \mathrm{e}^{(\alpha(x)-a(x)) / n} \frac{\beta^{2}(x)}{n^{2}} .
$$

Since $\alpha(x), \beta(x), a(x)$, and $b(x)$ are bounded and continuous, the sequence $\left\{Y_{n}\right\}$ satisfies all the conditions of (E1)-(E3). Therefore, Theorem 2.2 follows from Theorem 2.1.

\section{Acknowledgements}

The author is greatly indebted to Professor Zenghu Li for his supervision. Thanks are also due to the anonymous referee for helpful comments.

\section{References}

[1] Ethier, S. N. And Kurtz, T. G. (1986). Markov Processes. John Wiley, New York.

[2] Feller, W. (1951). Diffusion processes in genetics. In Proc. 2nd Berkeley Symp. Math. Statist. Prob., University of California Press, Berkeley, CA, pp. 227-246.

[3] Höpfner, R. (1985). On some classes of population-size-dependent Galton-Watson processes. J. Appl. Prob. 22, 25-36.

[4] Ispány, M., Pap, G. ANd ZuiJlen, M. C. A. (2005). Fluctuation limit of branching processes with immigration and estimation of the means. Adv. Appl. Prob. 37, 523-538.

[5] JiřInA, M. (1960). Stochastic branching processes with continuous-state space. Czech. Math. J. 8, $292-313$.

[6] JiŘına, M. (1969). On Feller's branching diffusion processes. Časopis. Pěst. Mat. 94, 84-90.

[7] Kawazu, K. and Watanabe, S. (1971). Branching processes with immigration and related limit theorems. Theory Prob. Appl. 16, 34-51.

[8] Klebaner, F. (1984). Geometric rate of growth in population-size-dependent branching processes. J. Appl. Prob. 21, 40-49.

[9] LI, Y. (2006). On a continuous-state population-size-dependent branching process and its extinction. J. Appl. Prob. 43, 195-207.

[10] LI, Y. (2009). Uniqueness of solutions of martingale problems with degenerate coefficients. Preprint. Available at http://www.paper.edu.cn/en/paper.php?serial_number=200901-298.

[11] LI, Z.-H. (2000). Orenstein-Uhlenbeck type processes and branching processes with immigration. J. Appl. Prob. 37, 627-634.

[12] LI, Z. (2006). A limit theorem for discrete Galton-Watson branching processes with immigration. J. Appl. Prob. 43, 289-295.

[13] LiPow, C. (1977). Limiting diffusions for population-size-dependent branching processes. J. Appl. Prob. 14, 14-24.

[14] Rosenkranz, G. (1985). Diffusion approximation of controlled branching processes with random environments. Stoch. Anal. Appl. 3, 363-377.

[15] Stroock, D. (1975). Diffusion processes associated Lévy generators. Z. Wahrscheinlichkeitsth. 32, $209-244$. 